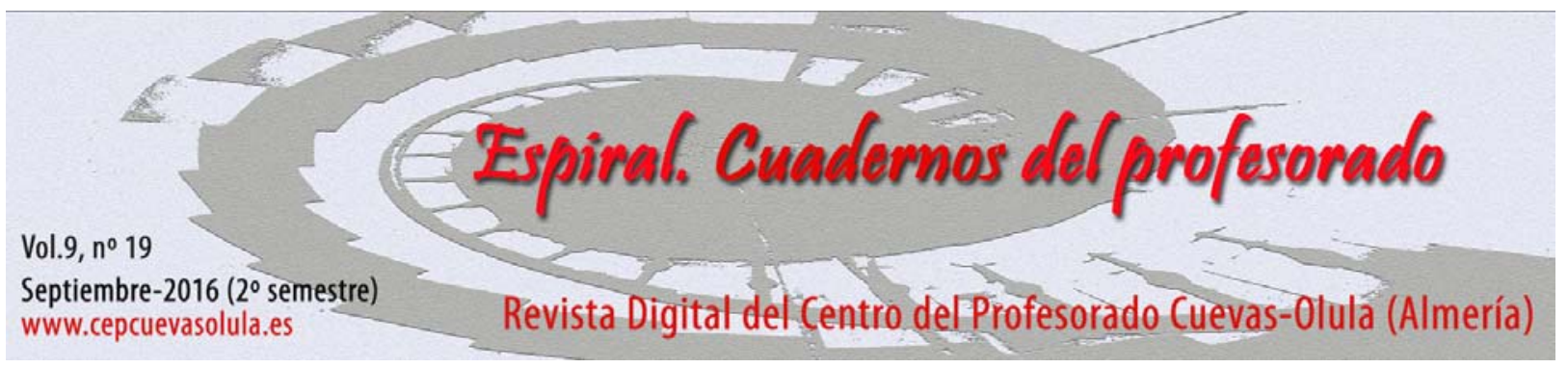

\title{
ENTORNO COMO REALIDAD DE APRENDIZAJE: PLANIFICACIÓN, ORGANIZACIÓN Y DESARROLLO DE SALIDAS ESCOLARES EN EDUCACIÓN INFANTIL
}

\author{
Use of the environment as a way for learning: planning, organization and development \\ of the school trips on the pre-school education
}

\section{David López-Ruiz y José Albaladejo Martínez}

Universidad de Murcia, España

\begin{abstract}
RESUMEN: El siguiente estudio tiene por objetivo ofrecer, a partir de la realidad educativa de un centro escolar público de nivel socioeconómico y cultural medio-bajo, un marco teórico-práctico que señale el valor de las salidas escolares como estrategias óptimas para utilizar el entorno natural, social y cultural como recurso. Dicho entorno recibe una especial atención desde el marco legislativo de la etapa de Educación Infantil por contribuir a la formación integral del alumnado. Por ello, es importante planificar las salidas, organizarlas y desarrollarlas teniendo en cuenta el contexto del centro y las posibilidades que éste ofrece. Así pues, a partir de los documentos institucionales del centro y las experiencias durante las prácticas escolares, se presenta una fundamentación teórica que incluye diferentes aspectos de las salidas escolares según el contexto socioeconómico y cultural del centro y la didáctica propia de una escuela del siglo XXI, y está complementada por unos ejemplos prácticos que pretendan mejorar el uso de estas estrategias y con ellas el aprendizaje del alumno y la calidad educativa. Para acabar, se extraen una serie de conclusiones que proponen mejoras en este ámbito, reflexionando a su vez sobre las salidas escolares como instrumento de compensación educativa.
\end{abstract}

Palabras clave: Entorno, salidas escolares; educación infantil; cultura; planes educativos.

ABSTRACT: The following study aims to provide, from the educational reality of a public culture mediumlow socioeconomic school and a theoretical and practical framework to bring the value of field trips and strategies best to use the natural, social and cultural environment as a resource. This environment receives special attention from the legislative framework of the pre-primary education to contribute to the integral formation of students. It is therefore important to plan outings, organize and develop given the context of the school and the possibilities it offers. So, from institutional documents of the center and the experiences during school practices, a theoretical foundation that includes different aspects of field trips is presented as the socioeconomic and cultural context of the school and self-teaching in a school of the century XXI, and to be supplemented by some practical examples that seek to improve the use of these strategies and these student learning and educational quality. Finally, a number of conclusions that propose improvements in this area, besides reflecting on field trips as an instrument of educational compensation are extracted.

Key words: Environment; field trips; Childhood Education; culture; educational plans.

López-Ruiz, M. y Albadalejo, J. (2016). Entorno como realidad de aprendizaje: Planificación, organización y desarrollo de salidas escolares en educación infantil. Espiral. Cuadernos del Profesorado, 9(19), 44-55. Disponible http://espiral.cepcuevasolula.es/

Fecha de recepción: 23/02/2016

Fecha de aceptación: 31/07/2016
Enviar correspondencia a: dlr@um.es 


\section{Introducción}

El entorno como realidad a través de la que se aprende resulta relevante en la educación actual de forma general, y en la etapa de Educación Infantil en particular (Pozo y Gómez, 2000). Los niños de hoy en día, por diversas causas (trabajo de ambos padres, carga excesiva de actividades extraescolares, dominio del mundo tecnológico en el tiempo recreativo, etc.) cada vez tienen menos oportunidades de interaccionar con su entorno próximo, es decir, de experimentar situaciones que les hagan comprender la realidad que está a su alrededor. Así, la escena antes común de los niños jugando en grupo, en la calle o en un parque, parece haberse perdido o en su lugar deteriorado. Ahora, el modo de juego tiende más al que se realiza de forma individual en casa con los medios tecnológicos, lo que hace al niño abstraerse de la realidad que sucede fuera de su casa.

Además, en un mundo tan globalizado se podría apreciar una perdida de la disposición a acercarse ya conocer lo que está a nuestro alrededor. En ocasiones no se es consciente del todo de que la identidad natural, socioeconómica y cultural del entorno próximo es fundamental para observar el resto de identidades del mundo y así poder abrir la mente hacia otras culturas y formas de vida. Todo esto puede posibilitar la formación de un sistema de valores basado en la tolerancia, la igualdad y el respeto (Bolívar, 2006).

Por ello, la escuela ha de compensar este déficit de experiencia en el entorno a través de las salidas, itinerarios didácticos y visitas que permitan al alumno dotar de significado al medio que le rodea. El problema surge cuando la escuela no ofrece lo suficiente dichas experiencias o las ofrece sin un sentido didáctico.

De este modo se considera que, al igual que autores como Vilarrasa (2003) o De Los Reyes (2009) se ha perdido ese espíritu explorador e innovador que se daba en clase por salir del centro, por ampliar horizontes. Para la época de la industrialización y posterior, el modelo de sistema escolar basado en clases instructivas dentro del aula no era pedagógico pero sí "práctico" para la sociedad que se estaba dando en ese momento, la cual demandaba mano de obra formada para un fin cerrado y mecánico. En la actualidad, los tiempos han cambiado mucho, y parece que no se ha avanzado (como en parte del sistema educativo) hacia una escuela del siglo XXI inmersa en una sociedad que ahora demanda personas con visión de mundo, personas realistas y con una mente abierta, personas que respeten lo que conocen, que convivan e interaccionen en un mismo entorno. Se ha "encerrado" a los alumnos en un aula para enseñarles la realidad a través de pictogramas, libros de texto o vídeos, cuando la auténtica la realidad es la que encuentran tras los muros del centro.

También es cierto que existen centros educativos donde se mantienen vivas algunas concepciones de la Escuela Nueva y otros movimientos pedagógicos del siglo XX que apuestan por un cambio en el enfoque escolar. Este enfoque está dirigido principalmente a poner al alumno en contacto con lo que aprende mediante estrategias como las salidas escolares. El contacto directo con la realidad aporta un gran significado en el pensamiento de los niños acercándolos a la importancia que tiene una salida escolar. ¿A cuántos niños no les cuesta dormir la noche de antes de una excursión? ¿Con qué disposición van ese día al colegio? Por lo que esto nos lleva a preguntarnos: ¿Y si aprovechamos ese entusiasmo y motivación que sienten los niños hacia las salidas escolares para hacer que conozcan mejor su entorno?

\section{Objetivo}

El objetivo en el que se centra este estudio consiste en reflejar la importancia de las salidas escolares en Educación Infantil analizando la realidad dada por el contexto socioeconómico y cultural, y aportando sobre ella un marco teórico-práctico que fundamente el análisis reflexivo para establecer unas propuestas de mejora en forma de conclusiones.

\section{La realidad de las salidas escolares en el centro educativo}

Puesto que el eje principal del tema son las salidas escolares, se han de reflejar aquellos aspectos del centro que muestran la realidad sobre el funcionamiento de las mismas. Para ello, podemos recurrir, por una parte, a los instrumentos de planificación institucionales del colegio que 
hagan alusión a nuestro objeto de estudio (Programaciones docentes, proyecto educativo y programación general anual). Y por otra parte, nos valdremos de la Memoria del Centro para ver los resultados y conclusiones de las actividades extraescolares y complementarias.

Entre los documentos institucionales de planificación del centro, empezaremos señalando la Programación general anual (PGA), ya que en ella se incluye el Proyecto educativo, cuyo apartado 2.3.3 tiene por título "Programación de las actividades complementarias y extraescolares". De este punto, nos interesa señalar el epígrafe "A" dedicado a las salidas por el entorno escolar, que dice así:

"Quedan autorizadas todas las salidas próximas al entorno escolar con duración inferior a 2 horas para la mejor adecuación de los contenidos académicos y nuestro ambiente socio-natural. Se pondrán en conocimiento del equipo Directivo antes de su realización. No son necesarias que figuren en la programación general anual".

En lo que respecta a las actividades complementarias que implican salir del aula, tales como "ir al cine", "ir a la plaza del ayuntamiento" o "salir al entorno próximo", hemos de señalar la ausencia de un tratamiento organizativo y didáctico desarrollado en fases o momentos que abarquen tanto el antes como el después de la actividad, y no sólo el momento de realizarla. Los alumnos se limitan a realizar las visitas ese mismo día sin un trabajo previo que vaya más allá de una charla en la asamblea el día de antes. Además, durante las salidas se convierten en participantes "sin participación", limitados a ver, oír y callar. En el momento de después de llevarse a cabo, el significado y los aprendizajes de la salida son prácticamente inexistentes ya que no se hay un trabajo posterior que vaya más allá de una asamblea para compartir opiniones.

Otro punto clave que ha de desarrollarse sobre la realidad del centro, en lo que respecta a las salidas escolares, son esas "salidas al entorno" a las que se refieren los documentos de planificación institucionales. En esta planificación no se tienen en cuenta las condiciones climatológicas óptimas para salir al entorno que suelen darse especialmente durante el segundo y tercer trimestre. No obstante, lo que más destaca es el desaprovechamiento de las posibilidades que ofrece el entorno del centro como recurso didáctico para trabajar muchos de los contenidos que se analizan en clase, pertenecientes a unidades didácticas, tales como "La calle", "La Primavera" o "Los oficios".

En síntesis, sobre la realidad del centro respecto a nuestro objeto de estudio podemos extraer las siguientes conclusiones:

- Las salidas extraescolares parecen no planificarse ni organizarse adecuadamente, de modo que algunas se venalteradas y otras directamente se cancelan.

- No se tienen en cuenta las posibilidades didácticas del entorno próximo a través de salidas, visitas o itinerarios.

- Las actividades extraescolares y complementarias fuera del aula carecen en su desarrollo de momentos o fases anteriores y posteriores a la salida, así como de una participación activa del alumnado durante la misma.

- Las actividades extraescolares no suelen adecuarse a las condiciones socioeconómicas de las familias, por lo que la participación del alumnado en ellas es muy baja.

A la vista de esta realidad respecto a las salidas escolares, se muestra a continuación una fundamentación teórica en la que se señala el valor legislativo y didáctico del entorno en Educación Infantil. En ella se indaga en las salidas escolares desde su conceptualización, tipos y fases hasta el marco normativo que las regula. Además se valora la función del profesor como participante en la propuesta de las salidas y se muestran algunas experiencias de salidas escolares. Finalmente se reflexiona sobre el valor de las salidas como instrumento de compensación educativa.

\section{Salidas escolares}

Partiendo de la idea de que los recursos por sí solos no educan, es importante aprovechar los recursos del entorno de una manera realmente didáctica. Para ello, se emplean estrategias de organización como las salidas, visitas de estudio, itinerarios y excursiones, en las que los maestros ponen a los niños en contacto con el medio (Aranda, 2003). Salir del aula utilizando estas estrategias supone, además de afianzar valores y normas de comportamiento como grupo, aprender a orientarse en 
el espacio, a descubrir elementos del paisaje que conforman la realidad (natural, social y cultural) y a racionalizar un espacio difícil de aprehender de golpe mediante los itinerarios (Escamilla, 2009).

Tanto es el valor que tiene salir del aula, que "se ha ido convirtiendo en signo y seña de renovación pedagógica" (Vilarrasa, 2003, p.13) al considerarse puente entre el aprendizaje y la experiencia del alumno. Desde este enfoque, De Los Reyes (2009, p. 8) afirma que:

"Desde la escuela activa (recordemos el valor otorgado a las excursiones escolares por la Institución Libre de Enseñanza en la España de la primera mitad del siglo XX) las salidas escolares siempre han estado ligadas a los movimientos más importantes de renovación pedagógica. Su propuesta responde a la idea que niños y niñas aprenden gracias a su experiencia personal directa y que la escuela debe facilitar que el alumno, por sí mismo y por inducción, descubra y construya los conocimientos: observa, busca, constata, recoge, manipula y explica lo que ve o descubre".

Es por ello que tal como asegura Olvera (1987, p. 111) "hemos de acostumbrarnos a una clase de puertas abiertas". Para ello, es importante superar las barreras organizativas y las limitaciones que tienden a atribuirse a esta etapa. Los alumnos de Educación Infantil probablemente sean los que más necesiten aprender del propio medio, dado que son los que menos oportunidades han tenido de interaccionar con él. Así pues, de lo expuesto anteriormente se puede considerar que utilizar el entorno como recurso a través de salidas, itinerarios didácticos y visitas puede ser una estrategia óptima para dotar de sentido a los aprendizajes al vincularlos a la experiencia. Además del aspecto motivador y lúdico que contienen las salidas escolares también poseen un enorme valor didáctico que desde el centro y el aula ha de saber aprovecharse. Este aprovechamiento, que repercute directamente en el estudiante se desarrolla con el objetivo de ofrecer una enseñanza ajustada a las necesidades del contexto y de los alumnos.

\section{Definiciones, características y tipos}

Para hablar sobre este tipo de estrategias, conviene previamente mostrar algunas definiciones de las mismas, así como los aspectos que las caracterizan y los diferentes tipos que proponen los autores. Advirtiendo la imprecisión de los campos que abarca cada uno de los términos, Delgado y Alarios (1994, p. 158) ofrecen las siguientes definiciones:

Salida: "Actividad que se realiza fuera del centro educativo con objeto de cubrir alguno de los objetivos del currículum y que por extensión puede aplicarse al conjunto de otras actividades" (itinerario, visita, paseo...).

Itinerario: "Actividad fuera del aula que de una manera más o menos estructurada dirige el interés del alumno hacia los elementos que se localizan a lo largo de un recorrido y su distribución".

Visita: "Actividad fuera del aula cuyo centro de interés se sitúa en un punto concreto_ museo, taller, monumento, institución, centro comercial, etc.".

Con respecto a esto, Delgado y Alarios (1994) añaden que dichas definiciones no ofrecen información sobre el aspecto didáctico, por lo que realizan una tipología de los itinerarios no suelen aparecer en "estado puro", produciéndose una gran cantidad de combinaciones, superposiciones y variantes entre ellos.

De este modo, por el contenido temático distinguen entre: "itinerarios temáticos" centrados en un aspecto concreto e "itinerarios disciplinares" donde se percibe la realidad como un todo. Según la metodología que se lleve a cabo en las salidas proponen: "itinerarios descriptivos" que se asemejan a guías turísticas; "itinerarios dirigidos" en los que se le pide participación puntual al individuo que lo realiza; "itinerarios inducidos" que nacen de una hipótesis para que el sujeto indague; e "itinerarios libres" en los que se explora el entorno desde un centro de interés. Por último, en función del tipo de actividades distinguen entre: "itinerarios de sensibilización"; "itinerarios de análisis-observación" e "itinerarios de diagnóstico" (Delgado y Alarios, 1994, pp. 159-160)

Olvera (1987, p. 112) define el itinerario como "la conjunción de diversos puntos los cuales están enlazados de una forma natural entre sí, en razón de ser o relacionar una misma unidad 
temática", mientras que Cuenca (2011) recoge los tipos de itinerarios que establece Insa (2002), distinguiendo: itinerarios referidos a la historia; itinerarios urbanos e interurbanos; itinerarios en relación a personajes ilustres; itinerarios tecnológicos e itinerarios naturales.

Respecto a las salidas, Estorch, Gómez, González, Quesada, y Quijano (1993, p.7) las definen como "experiencias educativas, que se realizan en grupo (con distintos agrupamientos) con los niños y niñas y la participación de los adultos y que implica un desplazamiento dirigido a otros espacios fuera o dentro del recinto escolar".

De esta última definición, nos interesa destacar, por un lado, el carácter social que tiene toda salida (sea del tipo que sea), ya que la agrupación que se organiza en dicha estrategia suele ser de gran grupo; y por otro lado, la posibilidad de considerar como salida, por ejemplo, un recorrido por el patio o una visita a la biblioteca del centro, donde el aprendizaje puede ser más significativo y real de lo que se espera.

Vilarrasa (2002, pp. 9-13), establece tres tipos de salida según el momento de la secuencia didáctica en la que se lleve a cabo: salida vivencial, salida de experimentación y salida de participación. A continuación presentamos un breve resumen de las tres, destacando de cada una la fase en la que se lleva a cabo y los objetivos que pretende:

La salida vivencial se desarrolla en la fase inicial de la secuencia y tiene por objetivos aumentar en los alumnos su rango de experiencias vividas en el entorno, de las cuales se partirán para desarrollar los contenidos en el aula; introducir y motivar al alumnado hacia un tema; y ponerlo en contacto directo con elementos, personas y realidades desconocidas.

La salida de experimentación, conocida como "la salida clásica" se lleva a cabo durante la fase de desarrollo del proceso didáctico. En ella se pretende introducir nuevos conocimientos acercando el aprendizaje a la experiencia del alumno a través de la experimentación y de la investigación-acción. Es importante señalar que requiere de un trabajo previo en la fase inicial, así como de un trabajo posterior en la fase de síntesis para que la salida tenga un sentido pleno y constructivo. Aunque este aspecto se detallará en próximos apartados.

Por último, la salida de participación se desarrolla en la fase de síntesis de la secuencia de enseñanza y se lleva a cabo con el propósito de extraer conclusiones sobre los aspectos trabajados durante las fases anteriores. Se pretende que los estudiantes reflexionen sobre la acción promoviendo de esta forma una serie de pautas de colaboración social que impliquen al alumno en el entorno que lo rodea.

Además, según Vilarrasa (2002), existen una serie de características que aportan a cualquier salida escolar la calidad suficiente para reconocer su valor didáctico debiendo ser: vivenciales, complejas, participativas, globales y compensatorias.

\section{Marco regulador de las salidas escolares}

Las salidas escolares que se lleven a cabo en un centro tienen que formar parte de la planificación institucional del mismo, a excepción de las salidas escolares al entorno próximo, que no se incluyen en la PGA. Su duración será inferior a 2 horas y tendrán que ser comunicadas previamente al equipo directivo. Sin embargo, al observar la doble denominación que reciben en los documentos legislativos e institucionales nos planteamos la siguiente cuestión: ¿Son las salidas escolares actividades extraescolares o se consideran actividades complementarias? La respuesta más acertada para esta cuestión no es cerrada, sino que parece llevar a la interpretación individual de cada caso para determinar la pertenencia a uno u otro grupo de actividades, dada la casuística del tema. Ésta parece ser una labor confusa, ya que para la Región de Murcia no existe regulación específica de estas actividades, a diferencia de otras Comunidades Autónomas.

No obstante, Morales (2014), ofrece una comparativa entre actividades complementarias y extraescolares, donde las mayores diferencias entre ambas residen en la voluntariedad (extraescolares) u obligatoriedad (complementarias) para realizarlas, ubicación horaria dentro (complementarias) o fuera (extraescolares) del horario escolar, y el carácter formal (complementarias) o no formal (extraescolares) de la educación que en ellas se imparte. Como decimos, habrá de interpretarse cada 
salida para entenderla bajo una u otra denominación. En cambio, algo que sí comparten tanto unas como otras es que ambas han de ser aprobadas por el Consejo Escolar y estar recogidas en la Programación General de Aula.

Llegados a este punto, cabe desarrollar brevemente el marco normativo que regula la planificación institucional del centro en la que se incluyen las actividades complementarias y extraescolares y aquellos aspectos que inciden directamente sobre ellas. Para ello, se hace referencia a la orden de 22 de septiembre de 2008, por la que se regulan, para la Comunidad Autónoma de la Región de Murcia, la implantación, el desarrollo y la evaluación en el segundo ciclo de la Educación Infantil (CARM, 2008, pp. 31234-31236), de acuerdo con el Real Decreto 1630/2006, de 29 de diciembre, por el que se establecen las enseñanzas mínimas del segundo ciclo de Educación Infantil (R.D, 1630/2006), y con el Decreto 254/2008, de 1 de agosto, por el que se establece el currículo del segundo ciclo de la Educación Infantil en la Comunidad Autónoma de la Región de Murcia (Decreto 254/2008).

En su artículo 3, la Orden 238/2008 expone que:

"Los centros docentes desarrollarán y completarán, en su caso, el currículo del segundo ciclo de la Educación Infantil establecido en el Decreto número 254/2008, de 1 de agosto, mediante programaciones docentes, adaptándolo a las características de los niños y a su realidad educativa. El resultado de esta concreción formará parte del Proyecto educativo del centro".

Más adelante, en el artículo 8, referido al Proyecto Educativo, se otorga a los centros docentes la función de situar la concreción de los currículos en su contexto social y cultural. Además, se determina cómo será el proceso de propuesta y aprobación del Proyecto Educativo, quedando reflejados los participantes y sus funciones de la siguiente forma:

"El equipo directivo elaborará el Proyecto educativo del centro de acuerdo con las directrices establecidas por el Consejo escolar y las propuestas realizadas por el Claustro de profesores, correspondiendo al Consejo escolar su aprobación. Para el establecimiento de dichas directrices se tendrán en cuenta las características del entorno escolar y las necesidades educativas de los alumnos. En los supuestos de revisiones periódicas y modificaciones posteriores se seguirá el mismo procedimiento señalado anteriormente".

Dentro de lo que debe incluir el Proyecto Educativo, por nuestro objeto de estudio, se destaca principalmente: a) El análisis de las características del entorno escolar y las necesidades educativas, que en función del mismo, ha de satisfacer; c) La adecuación de los objetivos generales del segundo ciclo de la Educación Infantil, al contexto socioeconómico y cultural del centro y a las características del alumnado; y d) La concreción del currículo, a través de las programaciones docentes

En este sentido, es preciso hacer un paréntesis para darle valor al medio socioeconómico y cultural dentro del sistema educativo, puesto que se puede considerar el sistema educativo como un subsistema del sistema sociocultural (Gairín, 1994).

¿Por qué es tan importante tener en cuenta el contexto socioeconómico y cultural en un centro escolar? De las manifestaciones adquiridas en las relaciones entre sistema escolar-sistema educativo, educación y sociedad, currículo y necesidades sociales que presenta Gairín (1994) podemos extraer tres argumentos para contestar a esta cuestión.

En primer lugar, porque prepara al alumno de cara a la realidad social que le espera fuera del colegio, como individuo que ha de adquirir unas normas y como trabajador que ha de realizar una aportación a la sociedad. En segundo lugar, porque transmite al alumno el patrimonio cultural que le rodea. $\mathrm{Y}$ en tercer lugar, porque desde su pluralidad aporta al individuo valores como la participación, la tolerancia o la igualdad.

Siguiendo con la Orden 238/2008, del artículo 9 nos interesa destacar, por un lado, la perspectiva globalizadora desde la que han de ser elaboradas las programaciones docentes por el equipo de maestros que conforman el equipo de ciclo, y por otro lado, especialmente, uno de los 
aspectos que deberán incluir en las programaciones: i) la propuesta de actividades complementarias y extraescolares.

Así pues, es evidente la presencia (a pesar de ser escasa) de las salidas escolares en forma de actividades complementarias y extraescolares en el marco normativo que rige la planificación institucional de los centros docentes. Del mismo modo, para que un centro realice una oferta educativa de calidad y acorde al contexto socioeconómico y cultural, se hace necesario un análisis previo completo y detallado que determine las características del mismo.

\section{El papel del profesorado en la las salidas escolares}

De todo lo anterior, valoramos especialmente la figura del profesor como pieza clave para impulsar actividades complementarias y extraescolares, mediante la propuesta que, en las programaciones docentes insertas en el Proyecto Educativo, se hace al Consejo Escolar. Por ello, consideramos que para proponer salidas escolares ajustadas al entorno socioeconómico y cultural del centro, el profesor ha de tener una adecuada formación con respecto al medio natural, social y cultural que rodea al centro escolar. Escamilla (2009), piensa que es totalmente necesario conocer el entorno en el que tanto profesor como alumnos se encuentran a fin de ser más consecuente con las propuestas de salidas planteadas.

Aranda (2003, p. 40), además, defiende que tanto para analizar el contexto en un Proyecto Educativo como para intervenir en la práctica docente, "el conocimiento del entorno necesita ser tanto más vivo y directo cuanto menor es la extensión del territorio que se considere como espacio de referencia". En este sentido, a la hora de conocer las características del contexto natural, social, económico o cultural podemos considerar las siguientes escalas, ordenadas de menor a mayor extensión, y por consiguiente, de mayor a menor profundización en su descripción: barrio o localidad donde radica el Centro; ciudad o comarca donde radica el Centro; provincia o entorno a similar escala, Comunidad autónoma; Estado; Unión Europea y escala mundial (Aranda, 2003).

Además de tener la responsabilidad de conocer adecuadamente el entorno del centro escolar, consideramos que el maestro de Educación Infantil ha de estar dispuesto a "abrir su aula", entendiendo los procesos de enseñanza-aprendizaje desde un marco más amplio donde el alumno aprende de lo que vive a partir de las experiencias fuera del aula que programa el profesor. Para ello, es importante que el profesor se implique en observar, analizar y reflexionar sobre la "didáctica del medio", es decir, las posibilidades que tiene el entorno como recurso para ser objeto y medio de aprendizaje para sus alumnos. Un profesor que no tenga la intención de investigar las posibilidades didácticas del medio, tendrá que valerse de otros medios que representen la realidad, nunca tan valiosas como la realidad misma.

Así pues, tanto la correcta elección de las salidas como su planificación y organización resultan vitales para que el alumno aprenda a través de lo que el entorno puede ofrecerle. Con todo ello cabe destacar que el profesor enseña al alumno a conocer el entorno de dos modos: llevando el medio al aula (como espacio), y trasladando el aula (como grupo de alumnos) al medio, a través de las salidas escolares.

\section{Fases y tareas dentro de las salidas escolares}

A la vista de la realidad observada respecto a la planificación, organización y desarrollo de las salidas durante las prácticas escolares, y teniendo en cuenta las competencias de las que dispone el profesor, se hace necesario en este trabajo dedicar un apartado a cómo se pueden llevar a cabo las salidas escolares. Un aspecto fundamental en este asunto son los momentos o fases que integren las diferentes salidas y las tareas que se desempeñen en cada uno de ellas. Con la intención de ofrecer un marco amplio y diverso, nos acercaremos a los modelos que plantean distintos autores al respecto. En este sentido, todos coinciden en segmentar las salidas en tres momentos, que se corresponden con los momentos de antes, durante y después de la salida.

La Tabla 1 presenta una síntesis de las aportaciones de varios autores acerca de las fases de una salida escolar y las tareas realizadas en cada momento. Es importante indicar que no se trata de mostrar cuál es el modelo correcto, sino de ofrecer diferentes opciones sobre cómo trabajar una salida 
escolar. Cada salida se puede ajustar mejor o peor a cada modelo, de modo que las características de un mismo modelo pueden ser idóneas para un tipo de salida y a la misma vez no ser adecuadas para otra, dada la multiplicidad del tipo de salidas. Los modelos son los siguientes:

Tabla 1.

Modelos de fases y tareas de salidas escolares.

\begin{tabular}{|c|c|c|c|}
\hline AUTOR & ANTES & DURANTE & DESPUÉS \\
\hline $\begin{array}{l}\text { Cuenca } \\
\text { (2011) }\end{array}$ & $\begin{array}{l}\text { Motivación inicial. } \\
\text { Detección de ideas previas. } \\
\text { Presentación de una situación } \\
\text { problemática. }\end{array}$ & $\begin{array}{l}\text { Orientación espacial. } \\
\text { Observación, análisis, } \\
\text { descripción y comparación de } \\
\text { los elementos. } \\
\text { Recogida de información } \\
\text { relevante en fichas o } \\
\text { cuadernillos. }\end{array}$ & $\begin{array}{l}\text { Ordenamiento y síntesis de } \\
\text { los datos recogidos. } \\
\text { Presentación de la } \\
\text { información en forma de } \\
\text { murales, dibujos, debates, } \\
\text { etc. }\end{array}$ \\
\hline
\end{tabular}

\section{Fase de preparación}

Resolución de aspectos técnicos (permisos, transporte, coste económico,...).

Preparación de contenidos, objetivos y actividades de la salida: consulta bibliográfica; preparación de la documentación a entregar al alumnado; realización previa

Delgado y Alarios del recorrido; establecimiento (1994) de paradas a efectuarse; control horario y concertación del contenido con asesores externos.

Preparación previa de la salida en el aula: introducción de pautas de observación y registro de datos; organización de los grupos y el material.
Fase de reflexión y trabajo en el aula

Ejercicios dirigidos a completar la visión obtenida en el transcurso de la salida (trabajo con recursos

\section{Fase de realización}

Práctica en un término medio entre la observación pasiva y la hiperactividad.

Uso del cuaderno de campo como instrumento de registro.

Profesor visible en todo momento. audiovisuales, puesta en común y resúmen de datos).

Establecimiento de relaciones con universos mayores, similares o diferentes.

Desarrollo de propuestas y toma de decisiones (juegos de simulación y de roles, mesas redondas y campañas escolares).

Resolución de problemas. Elaboración de productos e informes (paneles, mapas, exposición de dibujos).

Asamblea de recuerdo: diálogo sobre lo que han visto y valoración de la experiencia.

Escucha atenta a los maestros o monitores.

Preguntas sobre alguna inquietud.

Exposición de fotografías tomadas durante la salida.

Expresión de la experiencia en los diferentes lenguajes.

Realización del cuaderno de viaje.

Razonamiento y valoración sobre las conductas de los alumnos.

Realización de murales. 


\begin{tabular}{|c|c|c|c|}
\hline \multirow{12}{*}{$\begin{array}{c}\text { Urones y } \\
\text { Sánchez- } \\
\text { Barbudo } \\
\text { (1997) }\end{array}$} & Fase previa & Fase de realización & Fase posterior \\
\hline & Elección del lugar. & \multirow{11}{*}{$\begin{array}{l}\text { Poner a los niños tarjetas } \\
\text { indicadoras. } \\
\text { Hacer unas pocas paradas } \\
\text { concretas para explicar cosas } \\
\text { importantes. } \\
\text { Se realizan las actividades de } \\
\text { desarrollo previstas: } \\
\text { observación; comparación; } \\
\text { escucha de sonidos; recogida } \\
\text { de objetos y muestras; } \\
\text { realización de dibujos, juegos, } \\
\text { fotos y grabaciones de sonidos } \\
\text { e imágenes. } \\
\text { Tiempo libre. }\end{array}$} & \multirow{11}{*}{$\begin{array}{l}\text { Puesta en común. } \\
\text { Realizar diferentes } \\
\text { actividades conectadas con } \\
\text { la salida (comentar } \\
\text { fotografías; escuchar } \\
\text { grabaciones; comparar, } \\
\text { clasificar y ordenar objetos } \\
\text { recogidos; realizar murales } \\
\text { y dibujos; resolver fichas; } \\
\text { aprender canciones o } \\
\text { realizar actividades de } \\
\text { refuerzo). } \\
\text { Hacer actividades } \\
\text { complementarias (de } \\
\text { ampliación). } \\
\text { Hacer una evaluación } \\
\text { posterior todos juntos. }\end{array}$} \\
\hline & Visita previa del maestro. & & \\
\hline & $\begin{array}{l}\text { Recopilar información en torno } \\
\text { al lugar de estudio. }\end{array}$ & & \\
\hline & Programación de la salida. & & \\
\hline & Contar con el permiso y la & & \\
\hline & Coraboracion de los paares. & & \\
\hline & saben sobre el tema. & & \\
\hline & Motivar a los alumnos. & & \\
\hline & $\begin{array}{l}\text { Informarles de lo que van a } \\
\text { hacer. }\end{array}$ & & \\
\hline & Explicarles lo que deben llevar & & \\
\hline & & & \\
\hline \multirow{5}{*}{$\begin{array}{l}\text { De Los } \\
\text { Reyes } \\
(2009)\end{array}$} & $\begin{array}{l}\text { Evaluación inicial } \\
\text { Presentación de la }\end{array}$ & \multirow{5}{*}{$\begin{array}{l}\text { Recoger datos a través de los } \\
\text { sentidos. } \\
\text { Uso del cuaderno de campo, } \\
\text { con juegos y espacio para } \\
\text { dibujos. } \\
\text { Momentos de descanso. }\end{array}$} & $\begin{array}{l}\text { Volcado de la información } \\
\text { recogida. }\end{array}$ \\
\hline & estudiar. & & Corrección de los cuadernos \\
\hline & Organización del aula en & & de campo. \\
\hline & & & Reflexiones y conclusiones. \\
\hline & & & \\
\hline \multirow{6}{*}{$\begin{array}{l}\text { Vilarrasa': } \\
(2002)\end{array}$} & $\underline{\text { Fase inicial }}$ & Fase de desarrollo & $\underline{\text { Fase de síntesis }}$ \\
\hline & Definir y plantear el problema. & \multirow{5}{*}{$\begin{array}{l}\text { Recoger datos (dibujar, } \\
\text { fotografiar, preguntar...). }\end{array}$} & Tratar la información. \\
\hline & Detectar ideas previas. & & Formular conclusiones. \\
\hline & $\begin{array}{l}\text { Motivar el interés de los } \\
\text { alumnos. }\end{array}$ & & $\begin{array}{l}\text { Valoración y formulación de } \\
\text { alternativas. }\end{array}$ \\
\hline & Organizar la tarea. & & \multirow{2}{*}{$\begin{array}{l}\text { Elaboración y aplicación de } \\
\text { proyectos y pautas de } \\
\text { conducta. }\end{array}$} \\
\hline & $\begin{array}{l}\text { Establecer objetivos de } \\
\text { aprendizaje. }\end{array}$ & & \\
\hline
\end{tabular}

\section{Salidas escolares como instrumento de compensación educativa}

En ocasiones muchos de los alumnos viven en lugares alejados de la ciudad de referencia, por lo que prácticamente no están en contacto con el entorno más urbano, salvo en alguna visita puntual con sus familiares. Además, de los niños que sí viven en el entorno próximo al centro, muchos de sus padres trabajan ambos la jornada completa, lo que supone que los niños quedan a cargo de otros familiares, generalmente los abuelos. Esto conlleva que esos alumnos no disponen de experiencias suficientes en su entorno natural, social y cultural que les haga conocer y comprender el medio en el que viven. No todos los niños tienen la oportunidad de encontrarse con el entorno, ni todos pueden vivirlo del mismo modo.

Todo ello se resume en que entre los alumnos de una misma aula se dan desigualdades con respecto a las experiencias que han vivido en el entorno y los aprendizajes que éstas han supuesto. Así, una niña que vive en una casa situada en una pedanía no tiene el mismo bagaje de experiencias y conocimientos sobre el entorno que puede tener un niño que vive en el centro de la ciudad, en contacto

\footnotetext{
${ }^{1}$ De los tres tipos de salida que propone Vilarrasa (2002): salida vivencias, salida de experimentación y salida de participación, se presenta el segundo tipo por ser el más frecuente en un aula.
} 
a diario con parques, tiendas, plazas, etc. Por todo ello, la escuela, de nuevo como agente educador, ha de paliar las desigualdades que las condiciones socioeconómicas y culturales de la familia puedan generar. En este sentido, Olvera (1987) advierte de las desigualdades que se dan entre las clases sociales de una misma aula, ya que los códigos que se utilizan en ella corresponden con los de las clases dominantes. Por esta razón, es muy importante que el niño encuentre un medio escolar capaz de comunicar con su código y sus intereses. De lo que se trata, en definitiva, es de romper el aislamiento cultural al que se ve sometido el niño desde que nace en desigualdad con otros niños más privilegiados.

Así pues, todos los alumnos han de tener derecho a conocer su entorno, para así poder valorarlo y respetarlo, y con él a las personas que lo integran. A la vista de los efectos de la globalización y la multiculturalidad, la sociedad necesita personas que conozcan el contexto socioeconómico y cultural en el que viven, y que estén dispuestas a respetarlo y a mejorarlo para el bien de todos.

\section{Hacia unas salidas escolares más reales}

En este apartado final del desarrollo del trabajo, se pretende ofrecer una serie de conclusiones y valoraciones extraídas tras haber analizado el objeto de estudio teniendo en cuenta la realidad del centro, y haber fundamentado dicho análisis con un marco teórico que abarcase diferentes aspectos de las salidas escolares en un centro escolar público. Por ello, se muestran a continuación los aspectos del trabajo que se han ido explicitando y las reflexiones que de ellos han surgido.

En primer lugar, se ha tenido en cuenta la relevancia del entorno en la etapa de Educación Infantil. Analizando el currículo establecido para la etapa, (en nuestro caso el de la Región de Murcia). Pronto se percibe que el entorno es un componente fundamental del mismo, ya que está presente a lo largo del documento, desde los principios generales de la etapa hasta en contenidos de las diferentes áreas de aprendizaje. De una forma especial se hace referencia al área de "Conocimiento del entorno" destacando la visualización por parte de los estudiantes de algo tan obvio. El entorno se define como la realidad en la que se aprende y sobre la que se aprende considerándolo como un valioso recurso didáctico en el que fundamentar aspectos esenciales del currículo. De este modo, entenderlo como recurso supone concebirlo como un medio compuesto por un conjunto de factores ambientales de toda índole (natural, social, económico, cultural, etc.) que se incluyen en el proceso educativo con el fin de mejorar los aprendizajes de los alumnos al acercarlos de forma directa a la realidad. En este sentido, se observa que la puesta en práctica de salidas didácticas por el entorno contribuye a desarrollar una parte importante del currículo de Educación Infantil.

Las salidas escolares, entendidas en su globalidad, reciben una insuficiente valoración y atención desde el centro escolar y el propio aula, ya que no se considera del todo el valor didáctico que aportan para conocer el entorno y crear experiencias significativas para los alumnos. Desde hace varias décadas, se ha tendido a organizar una escuela de puertas para adentro, sin tener en cuenta que la auténtica realidad se encuentra fuera de los muros del colegio. Esa tendencia se ha mantenido hasta nuestros días a pesar de que la sociedad ya no demanda personas con aprendizajes mecánicos, sino individuos capaces de resolver situaciones reales a través de la indagación, el manejo de la información y por supuesto la creatividad. Es por todo esto que existe la necesidad de recuperar el espíritu explorador con sentido didáctico de las salidas escolares y además, ofrecer y favorecer las posibilidades idóneas para ello.

En este sentido, uno de los aspectos más relevantes a tener en cuenta para que una salida escolar tenga auténtico sentido para el alumnado es que se realice una adecuada planificación y organización desde el centro escolar a través de los documentos institucionales de planificación (planificaciones docentes, proyecto educativo y programación general anual). Es fundamental que se lleve a cabo la realización de un análisis del contexto que sea exhaustivo, real y ajustado a las características que rodean e influyen en el centro, para así poder ofrecer propuestas educativas de mayor calidad y significado que permitan al alumno aprender a través del medio natural, social y cultural más próximo. 
Por otra parte, en lo que se refiere a la planificación y organización de las salidas escolares, es necesaria una labor conjunta de todos los miembros de la Comunidad Educativa que participen en su propuesta, supervisión o aprobación. Todos reman en la misma dirección y esa dirección no puede ser otra que la de establecer en el centro y en las aulas experiencias valiosas que ayuden a los alumnos a desarrollarse íntegramente.

La figura del docente como impulsor de propuestas de actividades complementarias y extraescolares, tiene la responsabilidad de considerar las posibilidades del entorno para aprovecharlas por medio de las salidas. Es imprescindible disponer de un conocimiento lo más amplio posible del entorno natural, social y cultural que rodea al centro escolar y en caso de que no sea el más adecuado proponer ampliar el recorrido. Además, el docente como responsable de un aula tiene la labor de desarrollar las salidas escolares dotándolas de un sentido realmente didáctico, alejándose de ese "salir por salir" y de contenidos carentes de sentido pedagógico. Es cierto que esta planificación y estructuración supone dedicación, esfuerzo, tiempo, preparación, y sobre todo iniciativa por cambiar a mejor lo que se le ofrece al niño con la finalidad de que aprenda y se desarrolle como alumno, como ciudadano y como persona.

Por ello, en el desarrollo de una salida escolar es fundamental que no se entienda ésta como fin en sí misma, sino como parte del proceso de enseñanza y aprendizaje. Que se utilice para conocer de primera mano la realidad del entorno y vincularla a lo que se aprende en clase. En definitiva, se concibe el aprendizaje como un establecimiento constante de relaciones entre lo que ya sabe y lo que puede aprender del medio. Para ello hay que tener en cuenta los distintos momentos o fases que componen una salida programando y llevando a cabo no sólo la salida en sí, sino una serie de actividades anteriores que preparen al alumno ante lo que se va a encontrar. También son necesarias actividades posteriores que le hagan relacionar y afianzarlos aprendizajes previos y los nuevos adquiridos formando así un conocimiento teórico y práctico, es decir, lo que supone un conocimiento real.

Experiencias como salir al parque, visitar un museo o pasear por la calle demuestran el enorme valor didáctico que poseen las salidas escolares. A través de ellas, el alumno consigue darle significado al mundo que le rodea y alcanzar esa reapropiación del contexto que defiende Vilarrasa (2003). Bajo esta premisa, un alumno que esté aprendiendo en clase los elementos que componen la calle será capaz de hacer suyo ese conocimiento cuando lo vea en su entorno, cuando se siente en un banco, tire un papel al contenedor azul o cruce la carretera de la mano de un adulto por el paso de peatones. La escuela, como agente educador, tiene la posibilidad de ofrecer experiencias de la vida real al alumno para guiarlo en su camino hacia el aprendizaje del medio natural, social y cultural en el que vive. En sintonía con la idea de que el alumno ha de ser el propio constructor de su conocimiento se puede apreciar que el alumno aprende más de lo que vive que de lo que le enseñan. Todo ello lleva a considerar las salidas escolares como un instrumento de compensación educativa.

En conclusión, las salidas escolares, adecuadamente planificadas y organizadas desde el centro y desarrolladas por el docente tienen un alto valor didáctico que contribuye y ayuda a los alumnos a conocer el entorno natural, social y cultural en el que viven desde una perspectiva holística. Salir del aula es aprender porque salir del aula es vivir en la realidad.

\section{Referencias}

Aranda, A.M. (2003), Didáctica del conocimiento del medio social y cultural en Educación Infantil. Madrid: Síntesis.

Bolívar, A. (2006). Familia y escuela: dos mundos llamados a trabajar en común. Revista de Educación, 339.

Cuenca, J.M. (2011). Concepciones del alumnado en Educación Infantil para la comprensión del medio sociocultural. Papel de las experiencias y el aprendizaje lúdico. En M. P. Rivero (coord.). Didáctica de las Ciencias Sociales para Educación Infantil. (pp. 111-129). Zaragoza: Mira Editores.

De Los Reyes, J.L. (2009). Mi casa, mi calle, mi ciudad: experiencias sobre el espacio infantil en el Madrid histórico. Revista Terr@ Plural,3(1),9-27. 
Delgado, E. y Alarios, M.T. (1994). La interacción fuera del aula: itinerarios, salidas y paseos. Tabanque: Revista pedagógica (9), 155-178.

Escamilla, A. (2009). Las competencias en la programación de aula. Infantil y primaria (3-12 años). Barcelona: Graó.

Estroch, P., Gómez, M.D., González, E., Quesada, M.D., y Quijano, R. (1993). Módulo didáctico 3. Salimos y aprendemos [versión electrónica PDF]. Sevilla: Junta de Andalucía. Consejería de Educación y Ciencia.

Gairín, J. (1994). Los planteamientos institucionales. El Proyecto de Centro. En P. Darder y J. Gairín, (Coords.), Organización de centros educativos. Aspectos básicos. (pp. 99-126). Barcelona: Praxis.

Insa, Y. (2002). Itinerarios urbanos, recursos y materiales didácticos para enseñar la ciudad. Iber: Didáctica de las ciencias sociales, geografía e historia, (32), 89-95.

López Parra, J. (2008). Salidas escolares. Aportaciones a la educación. Revista digital Ciencia y Didáctica, 3 , $105-110$.

Morales, G. (2014). Los servicios complementarios y las actividades complementarias y extraescolares: una necesidad educativo-formativa. Avances en supervisión educativa, 22, 1-24.

Olvera, F. (1987). La investigación del medio en el aula. Madrid: Penthalon.

Pozo, I. y Gómez M.A. (2000). Aprender y Enseñar Ciencias. Del conocimiento cotidiano al conocimiento científico. Enfoques para la enseñanza de la ciencia. Madrid: Ediciones Morata.

Urones, C. y Sánchez-Barbudo, M. (1997). La organización de salidas al entorno en educación infantil. Revista Interuniversitaria de Formación del Profesorado, 1, 1-25.

Vilarrasa, A. (2003, abril), Salir del aula. Reapropiarse del contexto. Íber. Didáctica de las Ciencias Sociales, Geografía e Historia, 36, 13-25.

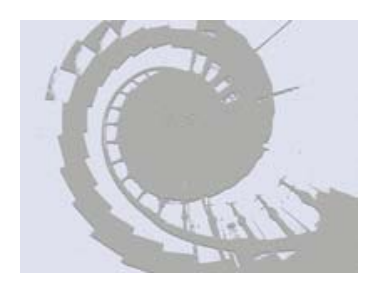

